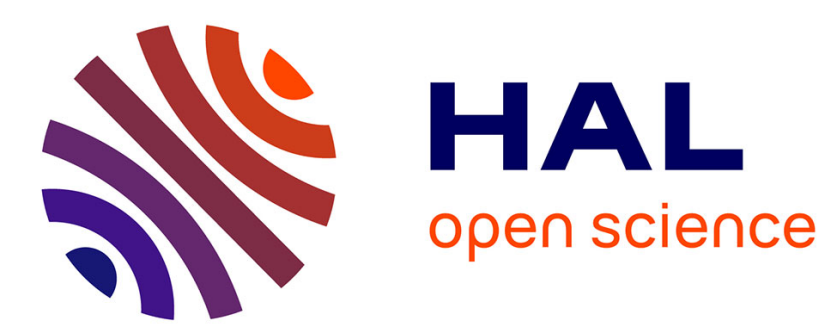

\title{
Dissipativeness and dissipativation of discrete-time switched linear systems
}

Marc Jungers, Francesco Ferrante, Jérôme Lohéac

\section{To cite this version:}

Marc Jungers, Francesco Ferrante, Jérôme Lohéac. Dissipativeness and dissipativation of discrete-time switched linear systems. CDC 2019 - 58th IEEE Conference on Decision and Control, Dec 2019, Nice, France. pp.5760-5765. hal-02194164

\section{HAL Id: hal-02194164 https://hal.science/hal-02194164}

Submitted on 20 Nov 2020

HAL is a multi-disciplinary open access archive for the deposit and dissemination of scientific research documents, whether they are published or not. The documents may come from teaching and research institutions in France or abroad, or from public or private research centers.
L'archive ouverte pluridisciplinaire HAL, est destinée au dépôt et à la diffusion de documents scientifiques de niveau recherche, publiés ou non, émanant des établissements d'enseignement et de recherche français ou étrangers, des laboratoires publics ou privés. 


\title{
Dissipativeness and Dissipativation of discrete-time switched linear systems
}

\author{
Marc Jungers, Member, IEEE, Francesco Ferrante, Member, IEEE, and Jérôme Lohéac
}

\begin{abstract}
Dissipativeness of dynamical systems is a crucial notion in control theory that consolidates the link with physics. It extends Lyapunov theory for autonomous systems to open ones and formalizes the relation between frequency domain conditions and matrix inequalities in state space representation. As emphasized in the limited and recent literature on this topic, dissipativeness of hybrid or continuous-time switched systems is a not intuitive and delicate notion. This paper copes with the dissipativeness analysis of discrete-time switched linear systems. Conditions in the form of linear matrix inequalities are provided to ensure dissipativeness of such systems with arbitrary switching law. The approach relies on modal storage functions. A second contribution is to design feedback switching laws, based on a min-switching strategy related to the modal storage functions, which ensures a dissipative behaviour of the closed-loop system. Implication in terms of passivity and stability of one single switched system, paving the way to the framework of interconnected switched sub-systems are discussed, before numerical illustrations.
\end{abstract}

\section{INTRODUCTION}

The notion of dissipativeness for dynamical systems has been formalized by Willems in [1] for continuous-time nonlinear systems. When considering a supply rate, a system is called dissipative if there exists a storage function associated with this supply rate. It can be interpreted as the fact that the system absorbs more energy from the external environ than it supplies. This concept extends Lyapunov theory, related to autonomous system, to the framework of open systems and has crucial implications for the interconnection of dynamical systems [1] and small gain theory. A large part of physical systems are dissipative as emphasized in [2]. That consolidates also the link between physics and control theory.

Independently, Yakubovich [3] and Kalman [4] obtained what is now known as the Kalman-Yakubovich-Popov (KYP) Lemma for continuous-time time-invariant linear systems, (or positive real lemma). This lemma allows one to establish an equivalent relation between linear matrix inequalities (concerning to the state space representation of linear systems) and a condition in the frequency domain. It was originally obtained to solve the Lur'e problem $[5,6]$. See also $[7,8]$ for a detailed discussion.

\footnotetext{
Marc Jungers and Jérôme Lohéac are with Université de Lorraine, CNRS, CRAN, F-54000 Nancy, France. Email: \{marc.jungers; jerome. loheac\}@univ-lorraine.fr

Francesco Ferrante is with Univ. Grenoble Alpes, CNRS, GIPSA-lab, F-38000 Grenoble, France. Email: francesco.ferrantelgipsa-lab.fr

Research funded in part by ANR via project HANDY, number ANR-18-CE40-0010.
}

The counterpart for discrete-time time-invariant systems has been provided in [9] and fills the gap between linear matrix inequalities weighted by the matrices of the state space representation and the harmonic responses in the frequency domain.

Dissipativeness of specific classes of systems has been studied (see for instance the references in [8]), yet the notion of passivity encounters a great attention in the literature. Passivity is a particular type of dissipativeness for systems with equal number of inputs and outputs, when considering a supply rate defined as the scalar product between the input and the output.

For switched systems, some preliminary results have been published several years ago, mainly in [10] and [11]. In the latter, it is shown that as for stability, the notion of passivity for switched system is not intuitive and underlines that switching among passive modes may destroy passivity.

The paper [12] presented a notion of passivity for switched systems with a multiple storage function approach. First the analysis of passivity is provided for a given switching law and secondly, under suitable conditions (involving localized form of KYP-Lemma and the $S$-procedure), a min-switching law strategy over the modal storage functions is proposed. In [13], dissipativity for continuous-time switched systems is investigated by considering multiple storage functions and multiple supply rates, including the concept of cross supply rate related to the link between active and inactive modes.

In [14], on the one hand, the link between passivity analysis of continuous-time switched systems and linear matrix inequalities and on the other hand, the link between the design of switching law to ensure the passivity of the resulting switched system and Yakubovitch-Metzler inequalities are provided.

The framework of discrete-time switched system has been investigated in [15]: the contribution deals with piecewise affine systems and provides first passivity analysis and also offers sufficient conditions in the form of linear matrix inequalities to design a switched state feedback to ensure closed-loop passivity.

It should be emphasized that in [16], passivity of nonlinear discrete-time switched systems switching suitably among passive and not passive modes is studied, but this paper assumes that there exists a common storage function. This is a very restrictive assumption.

Passivity for hybrid systems and more generally for the interconnection of hybrid sub-systems is more delicate and is discussed for instance in $[17,18]$. First contributions have been obtained when considering only weak notions of 
passivity: flow-passivity and jump-passivity, that is, when only the flow or jump is passive.

The contribution of this paper is focused on two main topics. The first study deals with the dissipativeness analysis of a discrete-time switched linear systems when considering an arbitrary switching law. The second contribution provides sufficient conditions to design of a min-switching law related to modal storage functions for the dissipativation of discrete-time switched linear systems. In both cases, we provide sufficient conditions in terms of linear matrix inequalities (LMIs) or parameterized ones.

This paper is organized as follows. In Section II, discrete-time switched linear systems are presented and the notion of dissipativeness with time-dependent supply rates and time-dependent storage functions is defined. Several closely related concepts of dissipativeness are discussed. Finally standard results in the frequency domain associated with the Kalman-Yakubovich-Popov (KYP) Lemma are recalled for linear time invariant systems. Section III is focused on the analysis of dissipativeness of discrete-time linear switched systems with arbitrary switching laws. Our main contribution consists of sufficient conditions in the form of linear matrix inequalities (LMIs) solving in a constructive way time-dependent supply rate and storage function for any switching law. Section IV is dedicated to design a min-switching law, which is a state feedback guaranteeing that the resulting system when such a switching law is applied, is dissipative. Discussions about sufficient conditions to ensure stability and stabilization of discrete-time switched systems are also provided. A numerical illustration emphasizes the applicability of the main contribution before concluding remarks.

Notation: Notation is quite standard. $\mathbb{R}$ denotes the set of real numbers, $\mathbb{Z}$ the set of integer ones, and $\mathbb{N}$ the set of nonnegative integer numbers. Moreover $\mathbb{N}^{*}=\mathbb{N} /\{0\}$ and $\mathbb{N}_{N}=\{1, \cdots, N\}$. The symbol $\star$ stands for a symmetric bloc and $\bullet$ is used as a shortcut such that $(\bullet)^{\top} M N=N^{\top} M N$, for suitable matrices $N$ and $M$, where $M^{\top}$ is the transpose of the matrix $M$ and $M^{*}$ its Hermitian. The set $\mathcal{M} \subset \mathbb{R}^{N \times N}$ of Metzler matrices is composed of matrices with nonnegative off-diagonal elements $\mathcal{M}:=\quad\left\{\Pi \in \mathbb{R}^{N \times N}, \pi_{j i} \geq 0, \forall(i, j) \in \mathbb{N}_{N}^{2}, i \neq j\right\}$. We define the subclass $\mathcal{M}_{\mathrm{d}}$ of $\mathcal{M}$ such that $\mathcal{M}_{\mathrm{d}}:=\quad\left\{\Pi \in \mathcal{M}, \pi_{i i} \geq 0, \sum_{\ell \in \mathbb{N}_{N}} \pi_{\ell i}=1, \forall i \in \mathbb{N}_{N}\right\}$. The convex combinations of positive definite matrices $P_{i}$ with weights being elements of the Metzler matrix $\Pi \in \mathcal{M}_{\mathrm{d}}$ is denoted as $(P)_{p, i}=\sum_{\ell \in \mathbb{N}_{N}} \pi_{\ell i} P_{\ell}$.

\section{DESCRIPTION OF THE SYSTEM AND DEFINITIONS}

Let us consider a discrete-time switched linear system, with $N \in \mathbb{N}^{*}$ modes satisfying $\forall k \in \mathbb{Z}$ :

$$
\begin{aligned}
x_{k+1} & =A_{\sigma(k)} x_{k}+B_{\sigma(k)} u_{k}, \\
z_{k} & =C_{\sigma(k)} x_{k}+D_{\sigma(k)} u_{k},
\end{aligned}
$$

with $x_{k} \in \mathbb{R}^{n}$ the state of the system, $u_{k} \in \mathbb{R}^{m}$ the exogenous input and $z_{k} \in \mathbb{R}^{p}$ the output. The switching law $\sigma: \mathbb{N} \rightarrow \mathbb{N}_{N}$ indicates at each time which mode is active.
Switched system (1)-(2) is by nature a time-varying system. It is of interest to notice that Willems in his fundamental paper has already considered the framework dissipativeness of nonstationary systems [1, Section 6]. Especially he pointed out that the supply rate, and also the storage function, may be time-varying. Here we adapt to the discrete-time domain the definitions by Willems.

Definition 1 (Willems Dissipativeness): A time-varying dynamical system (1)-(2) with a locally summable supply rate

$$
w: \mathbb{R}^{m} \times \mathbb{R}^{p} \times \mathbb{Z} \mapsto \mathbb{R}
$$

is said to be dissipative if there exists a nonnegative function $S: \mathbb{R}^{n} \times \mathbb{Z} \mapsto \mathbb{R}^{+}$, called storage function, such that, for any $\left(k_{0}, k_{1}, x_{k_{0}}\right) \in \mathbb{Z} \times \mathbb{Z} \times \mathbb{R}^{n}, k_{1} \geq k_{0}$, and any input $u_{k}$ defined over $\left\{k_{0} ; \cdots ; k_{1}\right\}$,

$$
S\left(x_{k_{0}}, k_{0}\right)+\sum_{k=k_{0}}^{k_{1}} w\left(u_{k}, z_{k}, k\right) \geq S\left(x_{k_{1}}, k_{1}\right),
$$

or equivalently,

$$
S\left(x_{k+1}, k+1\right)-S\left(x_{k}, k\right) \leq w\left(u_{k}, z_{k}, k\right),
$$

where $x_{k_{1}}$ is the state at time $k_{1}$ of the solution to system (1)-(2) when applying the control $u_{k}$. It is called lossless when the equality occurs in (4).

Several particular cases of dissipativeness can be pointed out, depending on the choice of supply rate, which can be viewed as the input power for the system [1].

- When considering a quadratic supply rate,

$$
\begin{aligned}
& w\left(u_{k}, z_{k}, k\right)=z_{k}^{\top} Q(k) z_{k}+2 z_{k}^{\top} L(k) u_{k}+u_{k}^{\top} R(k) u_{k}, \quad \text { (6) } \\
& \text { with } Q(k)=Q(k)^{\top} \in \mathbb{R}^{p \times p}, R(k)=R(k)^{\top} \in \\
& \mathbb{R}^{m \times m} \text { and } L(k) \in \mathbb{R}^{p \times m} \text {, we say that the system is } \\
& (Q(k), L(k), R(k)) \text {-dissipative. } \\
& \text { - When } Q(k)=-I_{p}, L(k)=0_{p \times m} \text { and } R(k)=\gamma^{2} I_{m},
\end{aligned}
$$
with $\gamma$ a scalar, the system has finite gain.

In addition, when $p=m$, i.e. when there are as many inputs as outputs, we use the following concepts:

- If $w\left(u_{k}, z_{k}, k\right)=z_{k}^{\top} u_{k}$, (that is $Q(k)=R(k)=0_{m}$ and $\left.L(k)=\frac{1}{2} I_{m}\right)$ the system is said passive.

- If $w\left(u_{k}, z_{k}, k\right)=z_{k}^{\top} u_{k}-\nu u_{k}^{\top} u_{k}$ with $\nu>0$, the system is said input strictly passive.

- If $w\left(u_{k}, z_{k}, k\right)=z_{k}^{\top} u_{k}-\nu z_{k}^{\top} z_{k}$ with $\nu>0$, the system is said output strictly passive.

Definition 1 involves a storage function. There are other definitions of dissipativeness for system, among them, Hill and Moylan considered the following definition by using only a condition about the supply rate [19] for the trajectories starting from the origin, that can be also extended to the time-varying case.

Definition 2 ( Hill \& Moylan's Dissipativeness): System (1)-(2) with a locally summable supply rate $w_{k}$, given by (3), is said to be dissipative if for any $k_{1} \geq k_{0}$, all admissible input $u_{k}$ defined over $\left\{k_{0} ; \cdots ; k_{1}\right\}$, any trajectory $x_{k}$ starting from $x_{k_{0}}=0$ satisfies: 


$$
\sum_{k=k_{0}}^{k_{1}} w\left(u_{k}, z_{k}, k\right) \geq 0 \text {. }
$$

Under some weak assumptions, which are given below, we can establish a link with the dissipativeness in the sense of Willems and provide necessary and sufficient conditions summarized in Theorem 1 for quadratic supply rates. This is a time-varying discrete-time counterpart of [19, Theorem 1].

Assumption 1: The state space of the system (1) is reachable from the origin. That is to say that given any $\left(x^{f}, k_{f}\right) \in \mathbb{R}^{n} \times \mathbb{Z}$, there exist $k_{i}=k_{i}\left(x^{f}, k_{f}\right) \in \mathbb{Z}$, $k_{i}<k_{f}$ and admissible control law $u_{k}=\tilde{u}_{k}\left(x^{f}, k_{f}\right)$ and switching rule $\sigma(k)=\tilde{\sigma}_{k}\left(x^{f}, k_{f}\right)$, that are defined over $k \in\left\{k_{i} ; \cdots ; k_{f}\right\}$, such that the corresponding state of (1) is steered from $x_{k_{i}}=0$ to $x_{k_{f}}=x^{f}$.

Assumption 2: For any $y \neq 0$, and $k \in \mathbb{Z}$, there exists some $u$ such that the supply rate (3) verifies $w(u, y, k)<0$.

Remark 1: Conditions to ensure the satisfaction of Assumption 1 have been provided in [20]. Here we assume those conditions hold. It should be noted that for discrete-time systems, as opposed to continuous-time ones, there may exist a bound related to the duration $k_{f}-k_{i}$. However this does not have any impact on the results. Assumption 1 concerns a structural property of the combination of the modes and is independent of the nature of the switching law (given, arbitrary or controlled). Assumptions 2 is only related to a suitable choice of matrices $Q(k), L(k)$ and $R(k)$ in the quadratic supply rate (6).

Theorem 1: System (1)-(2) is dissipative, in the sense of Definition 2, with respect to the quadratic supply rate given by (6) if and only if there exist a function $\phi: \mathbb{R}^{n} \times \mathbb{Z} \rightarrow \mathbb{R}$, which is quadratic with respect to its first argument, and real functions $\ell: \mathbb{R}^{n} \times \mathbb{Z} \rightarrow \mathbb{R}^{q}$ and $W: \mathbb{R}^{n} \times \mathbb{Z} \rightarrow \mathbb{R}^{q \times m}$ (for some integer $q$ ) satisfying

$$
\phi(x, k)>0, \text { for } x \neq 0 \text { and } \phi(0, k)=0
$$

and $\forall\left(x_{k}, u_{k}, k\right) \in \mathbb{R}^{n} \times \mathbb{R}^{m} \times \mathbb{Z}$, solution of the system (1)-(2),

$$
\begin{array}{r}
(\bullet)^{\top}[\bullet]^{\top}\left[\begin{array}{cc}
-H(k) & 0 \\
0 & H(k+1)
\end{array}\right] \boldsymbol{M}_{\sigma(k)}\left(\begin{array}{l}
x_{k} \\
u_{k}
\end{array}\right) \\
=(\bullet)^{\top}[\bullet]^{\top}\left[\begin{array}{cc}
R(k) & L(k) \\
\star & Q(k)
\end{array}\right] \boldsymbol{N}_{\sigma(k)}\left(\begin{array}{l}
x_{k} \\
u_{k}
\end{array}\right) \\
-\left\|\ell\left(x_{k}, k\right)+W\left(x_{k}, k\right) u_{k}\right\|^{2},
\end{array}
$$

where for any $i \in \mathbb{N}_{N}$,

$$
\boldsymbol{M}_{i}=\left[\begin{array}{cc}
I_{n} & 0_{n \times m} \\
A_{i} & B_{i}
\end{array}\right], \quad \mathbf{N}_{i}=\left[\begin{array}{cc}
0_{m \times n} & I_{m} \\
C_{i} & D_{i}
\end{array}\right],
$$

and $H(k)=\frac{1}{2} \frac{\partial^{2} \phi(x, k)}{\partial x^{2}}$ is half of the Hessian of the quadratic function $\phi$ at time $k$.

Remark 2: It should be mentioned that in the original result in the continuous-time domain [19, Theorem 1], the function $\phi$ is not assumed to be quadratic. This assumption is valid thanks to the result in [21] stating that for a dissipative linear system associated with a quadratic supply rate, any storage function can be represented as a quadratic function of the state. The time-varying assumption does not affect this result.

Proof: The proof follows the lines of [19, Theorem 1]. Sufficiency: Suppose that conditions (8)-(9) are verified, by rearranging the terms, we have

$$
\begin{aligned}
\sum_{k=k_{0}}^{k_{1}} w\left(u_{k}, z_{k}, k\right)= & \phi\left(x_{k_{1}+1}, k_{1}+1\right)-\phi\left(x_{k_{0}}, k_{0}\right) \\
& +\sum_{k=k_{0}}^{k_{1}}\left\|\ell\left(x_{k}, k\right)+W\left(x_{k}, k\right) u_{k}\right\|^{2} .
\end{aligned}
$$

Taking $x_{k_{0}}=0$, we conclude that (7) holds.

Necessity: Let introduce $\check{z}_{k}=z\left(x_{k_{0}},\left\{u_{\ell} ; \sigma(\ell)\right\}_{k_{0} \leq \ell \leq k}, k\right)$ denoting the value of the output $z_{k}$ at time $k$ related to the trajectory starting at $k_{0}$ from $x_{0}$ and when the input control and switching law $\left\{u_{\ell} ; \sigma(\ell)\right\}_{k_{0} \leq \ell \leq k}$ are applied. Assuming that condition (7) holds, we show first that the available storage function $\phi_{\mathrm{a}}\left(x_{k}, k\right)$ defined by

$$
\phi_{\mathrm{a}}\left(x_{0}, k_{0}\right)=\sup _{\substack{K \geq k_{0},\left\{u_{k} ; \sigma(k)\right\}_{k_{0} \leq k \leq K}}}-\sum_{k=k_{0}}^{K} w\left(u_{k}, \check{z}_{k}\right),
$$

is well defined and solves (8)-(9).

Independently of the sequence of input control and switching law $\left\{u_{\ell} ; \sigma(\ell)\right\}_{k_{0} \leq \ell}$ and by using Assumption 1, we can determine a time $k_{i}<k_{0}$ and admissible input and switching laws, defined over $\left\{k_{i}, \cdots, k_{0}-1\right\}$, such that $x_{k_{i}}=0$ can be steered to $x_{0}$ at $k=k_{0}$. We denotes with some abuse of notation, $\tilde{u}_{k}$ and $\tilde{z}_{k}$ the resulting input and output of this trajectory. Condition (7) implies

$$
-\sum_{k=k_{0}}^{K} w\left(u_{k}, z_{k}, k\right) \leq \sum_{k=k_{i}}^{k_{0}-1} w\left(\tilde{u}_{k}, \tilde{z}_{k}, k\right)<+\infty
$$

where the righthand side is an implicit function of the initial state $x_{0}$. The available storage function $\phi_{\mathrm{a}}$ defined by (11) is finite. In addition, thanks to Assumption 2, $\phi_{\mathrm{a}}$ is positive definite. By following the lines of [1], the available storage function is a solution to (4). To end the proof, we need to determine $\ell\left(x_{k}, k\right)$ and $W\left(x_{k}, k\right)$ such that for $\phi=\phi_{\mathrm{a}}$, conditions (8)-(9) are satisfied. Introducing $d\left(x_{k}, u_{k}, k\right)=\phi_{\mathrm{a}}\left(x_{k+1}, k+1\right)-\phi_{\mathrm{a}}\left(x_{k}, k\right)+w\left(u_{k}, z_{k}, k\right)$, we have $d\left(x_{k}, u_{k}, k\right) \geq 0$ thanks to (5) applied on $\phi_{\mathrm{a}}$. Hence, $\phi_{\mathrm{a}}$ and $w$ being quadratic functions, and $x_{k+1}$ and $z_{k}$ being affine in the input $u_{k}$, we can factorize $d\left(x_{k}, u_{k}, k\right)$ as $d\left(x_{k}, u_{k}, k\right)=\left\|\ell\left(x_{k}, k\right)+W\left(x_{k}, k\right) u_{k}\right\|^{2}$, with $\ell\left(x_{k}, k\right) \in$ $\mathbb{R}^{q}$ and $W\left(x_{k}, k\right) \in \mathbb{R}^{q \times m}$ for some integer $q$. That leads to conditions (8)-(9).

The framework of Theorem 1 is close to the one in [16] related also to switched discrete-time systems but that assumes only a unique supply rate and storage function. It extends furthermore to time-dependency the continuous-time result provided in [19, Theorem 1].

In the next sections, sufficient conditions will be provided to derive Theorem 1 in a constructive way in distinct frameworks. To end this preliminary section, we recall well established results for the dissipativeness of a discrete-time 
linear time-invariant (LTI) system, namely

$$
\begin{aligned}
x_{k+1} & =A x_{k}+B w_{k}, \\
z_{k} & =C x_{k}+D w_{k},
\end{aligned}
$$

with state $x_{k} \in \mathbb{R}^{n}$, exogenous input $w_{k} \in \mathbb{R}^{m}$, and output $z_{k} \in \mathbb{R}^{p}$. The transfer function of this system is denoted by $T(q)=C\left(q I_{n}-A\right)^{-1} B+D$, where $q \in \mathbb{C}$.

Considering system (12)-(13) and quadratic $(Q, L, R)$-dissipativeness, without lack of generality, it is possible to look for quadratic storage functions. The dissipative inequality (5) is then equivalent to the LMI in a symmetric matrix $P \in \mathbb{R}^{n \times n}$ such that

$$
\begin{aligned}
{[\bullet]^{\top}\left[\begin{array}{cc}
-P & 0 \\
0 & P
\end{array}\right]\left[\begin{array}{cc}
I_{n} & 0_{n \times m} \\
A & B
\end{array}\right] \leq } \\
{[\bullet]^{\top}\left[\begin{array}{cc}
R & L \\
\star & Q
\end{array}\right]\left[\begin{array}{cc}
0_{m \times n} & I_{m} \\
C & D
\end{array}\right] . }
\end{aligned}
$$

If (14) is verified, a storage function for system (12)-(13) is given by $S(x)=x^{\top} P x$, which is a solution to the time invariant dissipative inequality $S\left(x_{k+1}\right)-S\left(x_{k}\right) \leq$ $w\left(u_{k}, z_{k}\right)$. When considering the particular case of passivity, LMI (14) reads (see for example [9, Lemma 3]):

$$
\left[\begin{array}{cc}
A^{\top} P A-P & A^{\top} P B-C^{\top} / 2 \\
\star & B^{\top} P B-\left(D+D^{\top}\right) / 2
\end{array}\right] \leq 0_{n+m} .
$$

The existence of a matrix $P$ solution to LMI (14), without assumption on its definiteness is related to a frequency domain criterion, called circle criterion and mentioned in the following lemma (see the discussion in [7]).

The advantage of the frequency domain criterion in the KYP-Lemma lies in the fact that it can be numerically checked without the computation of the given matrix $P$, and as a consequence answer to the dissipative issue of a LTI system.

Lemma 1: Suppose that the pair $(A, B)$, of the system (12)-(13), is controllable. The two following conditions are equivalent:

1) For all $\omega \in \mathbb{R}$, $i \omega$ not eigenvalues of matrix $A$,

$$
0_{m} \leq\left(\begin{array}{c}
I_{m} \\
T\left(e^{i \omega}\right)
\end{array}\right)^{*}\left[\begin{array}{cc}
R & L \\
\star & Q
\end{array}\right]\left(\begin{array}{c}
I_{m} \\
T\left(e^{i \omega}\right)
\end{array}\right) ;
$$

2) There exists a symmetric matrix $P \in \mathbb{R}^{n \times n}$ such that LMI (14) is verified.

Lemma 1 calls several comments and remarks.

Remark 3: When considering strict inequalities (14) and (16), the equivalence between the two items is true even if the controllability assumption is relaxed.

Remark 4: In practice, in order to have a positive definite storage function, $P$ in (14) will be assumed to be positive definite. That will be assumed in the following.

Remark 5: Feasibility of the LMI (14) in the variable $P$ allows a time independent solution of relation (9), in which the choice of $\ell\left(x_{k}, k\right)$ and $W\left(x_{k}, k\right)$ is made to reach the equality.

Remark 6: The $(Q, L, R)$-dissipativeness with $P^{\top}=$ $P>0_{n}$ and $C^{\top} Q C \leq 0_{n}$, guarantees stability of system (12). This implication comes from the fact that the left-upper block in matrix inequality (14) reads $A^{\top} P A-$ $P<C^{\top} Q C$. This is obviously true when considering the particular case of passivity, because $C^{\top} Q C=0_{m}$.

\section{DISSIPATIVENESS OF DISCRETE-TIME SWITCHED SYSTEMS WITH ARBITRARY SWITCHING LAW}

In this section, we consider the system (1)-(2), with the assumption that the switching law $\sigma(\cdot)$ is arbitrary. Sufficient conditions to ensure that this system is dissipative irrespective to the choice of the switching law are provided in Theorem 2. The idea is to take advantage of the time-varying nature of the conditions in Theorem 1 to deal with the switching behaviour of the system (1)-(2). Nevertheless, due to the restriction of the time-dependency via the switching law, we provide only sufficient conditions. The conservatism of this is still an open issue. The obtained sufficient conditions, based on LMIs, allow to build a switching storage function related to a switching supply rate. This is an extension of [16].

Theorem 2: Consider the system (1)-(2) and $N$ triplets of matrices $\left(Q_{i}, L_{i}, R_{i}\right) \in \mathbb{R}^{p \times p} \times \mathbb{R}^{p \times m} \times \mathbb{R}^{m \times m}$ with $Q_{i}=Q_{i}^{\top}$ and $R_{i}=R_{i}^{\top}$. If there exist $N$ symmetric positive definite matrices $P_{1}, \cdots, P_{N}$ such that $\forall(i, j) \in \mathbb{N}_{N}^{2}$ the following LMIs are verified

$$
[\bullet]^{\top}\left[\begin{array}{cc}
-P_{i} & 0 \\
0 & P_{j}
\end{array}\right] \boldsymbol{M}_{i}<[\bullet]^{\top}\left[\begin{array}{cc}
R_{i} & L_{i} \\
\star & Q_{i}
\end{array}\right] \boldsymbol{N}_{i},
$$

with $M_{i}$ and $\boldsymbol{N}_{i}$ defined by (10), then the system (1)-(2) is dissipative with respect to a time-varying, or switching, quadratic supply rate $w\left(u_{k}, z_{k}, k\right)=w_{\sigma(k)}\left(u_{k}, z_{k}\right)$, with

$$
w_{i}\left(u_{k}, z_{k}\right)=z_{k}^{\top} Q_{i} z_{k}+2 z_{k}^{\top} L_{i} u_{k}+u_{k}^{\top} R_{i} u_{k}, \forall i \in \mathbb{N}_{N},
$$

and switching storage function $S\left(x_{k}, k\right)=x_{k}^{\top} P_{\sigma(k)} x_{k}$.

Proof: The proof is straightforward by considering $(i, j)=(\sigma(k), \sigma(k+1))$ and multiplying the LMI (17)

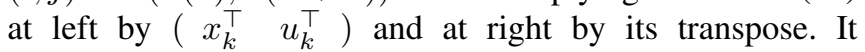
results that $S\left(x_{k}, k\right)=x_{k}^{\top} P_{\sigma(k)} x_{k}$ is positive definite and $x_{k+1}^{\top} P_{\sigma(k+1)} x_{k+1}-x_{k}^{\top} P_{\sigma(k)} x_{k} \leq w_{\sigma(k)}\left(u_{k}, z_{k}\right)$.

LMIs (17) with $i=j$ recovers condition (14) for discrete-time LTI system. As a consequence, each mode $i \in \mathbb{N}_{N}$ of system (1)-(2) is a LTI system which is $\left(Q_{i}, L_{i}, R_{i}\right)$-dissipative with respect to the supply rate $w_{i}\left(u_{k}, z_{k}\right)$ and modal (time independent) storage function $S\left(x_{k}, k\right)=x_{k}^{\top} P_{i} x_{k}$.

Remark 7: When Theorem 2 applies, thanks to the positive definiteness of matrices $P_{i}, i \in \mathbb{N}_{N}$, if $C_{i}^{\top} Q_{i} C_{i} \leq$ $0_{n}$, for all $i \in \mathbb{N}_{N}$, then the first diagonal block of LMI (17) reads $A_{i}^{\top} P_{j} A_{i}-P_{i}<0_{n}$, for any $(i, j) \in \mathbb{N}_{N}^{2}$, which ensures the stability of the discrete-time switched autonomous system $x_{k+1}=A_{\sigma(k)} x_{k}$ under any switching rule $\sigma(\cdot)$ [22].

When considering passivity as a specific dissipativeness, Theorem 2 applies with LMIs (17) replaced by the LMIs

$$
\left[\begin{array}{cc}
A_{i}^{\top} P_{j} A_{i}-P_{i} & A_{i} P_{j} B_{i}-C_{i} / 2 \\
\star & B_{i}^{\top} P_{j} B_{i}-\left(D_{i}+D_{i}^{\top}\right) / 2
\end{array}\right]<0_{m+n} .
$$




\section{SWITCHING LAW DESIGN FOR DISSIPATIVATION OF DISCRETE-TIME SWITCHED SYSTEMS}

This section is devoted to offer sufficient conditions allowing the dissipativation of a discrete-time linear switched system of the form (1)-(2), that is to say the existence of a switching rule making the system dissipative. Furthermore, under these sufficient conditions, it is possible to explicitly build and exhibit such a switching rule. The main result is gathered in the following theorem.

Theorem 3: Consider the system (1)-(2) and $N$ triplets of matrices $\left(Q_{i}, L_{i}, R_{i}\right) \in \mathbb{R}^{p \times p} \times \mathbb{R}^{p \times m} \times \mathbb{R}^{m \times m}$ with $Q_{i}=Q_{i}^{\top}$ and $R_{i}=R_{i}^{\top}$. If there exist $N$ symmetric positive definite matrices $P_{i}, i \in \mathbb{N}_{N}$ and a Metzler matrix $\Pi \in \mathcal{M}_{d}$ such that for any $i \in \mathbb{N}_{N}$ the following matrix inequalities are verified:

$$
[\bullet]^{\top}\left[\begin{array}{cc}
-P_{i} & 0 \\
0 & (P)_{p, i}
\end{array}\right] \boldsymbol{M}_{i}<[\bullet]^{\top}\left[\begin{array}{cc}
R_{i} & L_{i} \\
\star & Q_{i}
\end{array}\right] \boldsymbol{N}_{i},
$$

with $\boldsymbol{M}_{i}$ and $\boldsymbol{N}_{i}$ defined by (10), then system (1)-(2) is rendered dissipative by applying the min-switching rule related to the multiple storage functions

$$
\sigma(k)=\mu\left(x_{k}\right) \in \arg \min _{i \in \mathbb{N}_{N}} x_{k}^{\top} P_{i} x_{i} .
$$

Proof: Assume that the condition (19) is verified. Let

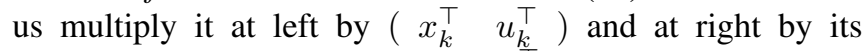
transpose, that yields $\left(A_{i} x_{k}+B_{i} u_{k}\right)^{\top}(P)_{p, i}\left(A_{i} x_{k}+B_{i} u_{k}\right)-$ $x_{k}^{\top} P_{i} x_{k} \leq w_{i}\left(u_{k}, C_{i} x_{k}+D_{i} u_{k}\right)$. Let us introduce the minimum storage function as $S_{\min }(x)=\min _{i \in \mathbb{N}_{N}} x^{\top} P_{i} x$, $\forall x \in \mathbb{R}^{n}$. Thus, we have

$$
\begin{aligned}
S_{\min }\left(A_{i} x_{k}+B_{i} u_{k}\right) & =\min _{j \in \mathbb{N}_{N}}(\bullet)^{\top} P_{j}\left(A_{i} x_{k}+B_{i} u_{k}\right), \\
& \leq(\bullet)^{\top}(P)_{p, i}\left(A_{i} x_{k}+B_{i} u_{k}\right), \\
& \leq x_{k}^{\top} P_{i} x_{k}+w_{i}\left(u_{k}, C_{i} x_{k}+D_{i} u_{k}\right),
\end{aligned}
$$

because the minimum of a set of positive scalars is always less or equal to any of their convex combinations. By selecting the min-switching strategy defined by (20), it yields

$$
\begin{aligned}
& S_{\min }\left(A_{\mu\left(x_{k}\right)} x_{k}+B_{\mu\left(x_{k}\right)} u_{k}\right)-S_{\min }\left(x_{k}\right) \\
& \leq w_{\mu\left(x_{k}\right)}\left(u_{k}, C_{\mu\left(x_{k}\right)} x_{k}+D_{\mu\left(x_{k}\right)} u_{k}\right) .
\end{aligned}
$$

The positive definiteness of $S_{\min }(\cdot)$ is inherited by the positive definiteness of matrices $P_{i}$, in finite number. The function $S_{\min }(\cdot)$ is thus the induced storage function related to the min-switching strategy (20).

It should be noticed that the min-switching rule, when the matrices $P_{i}$ are given, is only a (non-linear) state-feedback and does not depend explicitly on the supply rates $w_{i}\left(u_{k}, z_{k}\right)$ defined by (18). This choice may be done irrespectively with the input $u_{k}$.

Remark 8: With $C_{i}^{\top} Q_{i} C_{i} \leq 0_{n}$, the left-upper block of inequality (19) leads to the Lyapunov-Metzler inequalities $A_{i}^{\top}(P)_{p, i} A_{i}-P_{i}<0_{n}$, which is a sufficient condition to stabilize the autonomous system $x_{k+1}=A_{\sigma(k)} x_{k}$ via the min-switching strategy (20) (see [23] or [24,25] for more details). Less conservative implications may be investigated in future research.
Remark 9: The result in Theorem 3 deals with switched systems having not necessarily $\left(Q_{i}, L_{i}, R_{i}\right)$-dissipative modes. Nevertheless the $\left(Q_{i}, L_{i}, R_{i}\right)$-dissipativeness of the LTI system

$$
\begin{aligned}
x_{k+1} & =\pi_{i i}^{1 / 2} A_{i} x_{k}+\pi_{i i}^{1 / 2} B_{i} u_{k}, \\
y_{k} & =C_{i} x_{k}+D_{i} u_{k},
\end{aligned}
$$

is a required condition for the feasibility of inequalities (19), because it can be rewritten as

$$
\begin{array}{r}
{[\bullet]^{\top}\left[\begin{array}{cc}
-P_{i} & 0 \\
0 & \pi_{i i} P_{i}
\end{array}\right] \boldsymbol{M}_{i}<[\bullet]^{\top}\left[\begin{array}{cc}
R_{i} & L_{i} \\
\star & Q_{i}
\end{array}\right] \boldsymbol{N}_{i}} \\
-\sum_{j \in \mathbb{N}_{N}, j \neq i}[\bullet]^{\top}\left[\begin{array}{cc}
0 & 0 \\
0 & \pi_{j i} P_{j}
\end{array}\right] \boldsymbol{M}_{i} .
\end{array}
$$

One further necessary condition for the feasibility of inequalities (19) is that $R_{i}+L_{i} D_{i}+D_{i}^{\top} L_{i}^{\top}+D_{i}^{\top} Q_{i} D_{i} \leq 0_{m}$. That can be viewed by deriving the lower right block of inequality (19) and noticing that $B_{i}^{\top}(P)_{p, i} B_{i} \leq 0_{n}$.

It is noteworthy that our framework using multiple storage functions leads to a storage function, which apparently does not depend on the switching rule, however a main difference with the approach using a common storage function, as in [16] is that $S_{\min }(x)$ may not be differentiable with respect to $x \in \mathbb{R}^{n}$.

\section{NUMERICAL ILLUSTRATION}

This section provides a numerical example illustrating the contribution in Section IV. Let us consider the following example with $n=3, m=p=1$ and two modes $(N=2)$, resulting from the discretization, with an unitary sampling period, of exemple [14, Exemple 2].

$$
\begin{aligned}
& {\left[\begin{array}{r:c}
A_{1} & B_{1} \\
\hdashline C_{1} & D_{1}
\end{array}\right]=\left[\begin{array}{rrr:r}
0.82 & 0.59 & 0.20 & 0.09 \\
-0.41 & 0.01 & 0.18 & 0.20 \\
-0.36 & -1.12 & -0.36 & 0.18 \\
\hdashline-1 & 0 & 0 & 0.25
\end{array}\right] ;}
\end{aligned}
$$

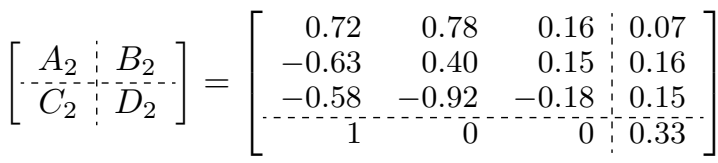

and the quadratic supply rates defined by $\left(Q_{1}, L_{1}, R_{1}\right)=$ $\left(Q_{2}, L_{2}, R_{2}\right)=(0,1 / 2,0)$. The two modes are stable, but are not passive. That can be checked numerically because the frequency domain inequality (16) is not satisfied, as shown in Figure 1, with frequency $\omega \in[-\pi, \pi]$ and $q=e^{j \omega}$.

Nevertheless, there is a solution to inequalities (19) with

$$
\begin{gathered}
P_{1}=\left[\begin{array}{rrr}
9.22 & -0.66 & 6.18 \\
-0.66 & 1.78 & -1.73 \\
6.18 & -1.73 & 5.77
\end{array}\right] ; \\
P_{2}=\left[\begin{array}{rrr}
2.25 & 0.07 & -0.03 \\
0.07 & 3.26 & 0.63 \\
-0.03 & 0.63 & 0.20
\end{array}\right] ; \quad \Pi=\left[\begin{array}{cc}
0 & 0.8 \\
1 & 0.2
\end{array}\right] .
\end{gathered}
$$

Let us consider as exogenous input $u_{k}=4 \sin (k)$. The min-switching strategy $\sigma(k)$ defined by (20) is depicted on Figure 2. This figure emphasizes also that the dissipative inequality (21) is verified, as expected. 


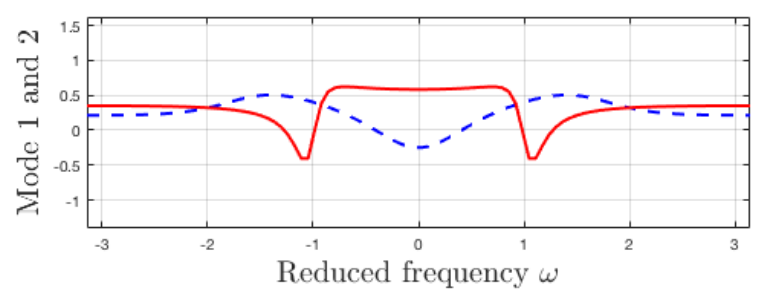

Fig. 1. Frequency domain inequality (16) for mode 1 (blue dashed line), and mode 2 (red line).
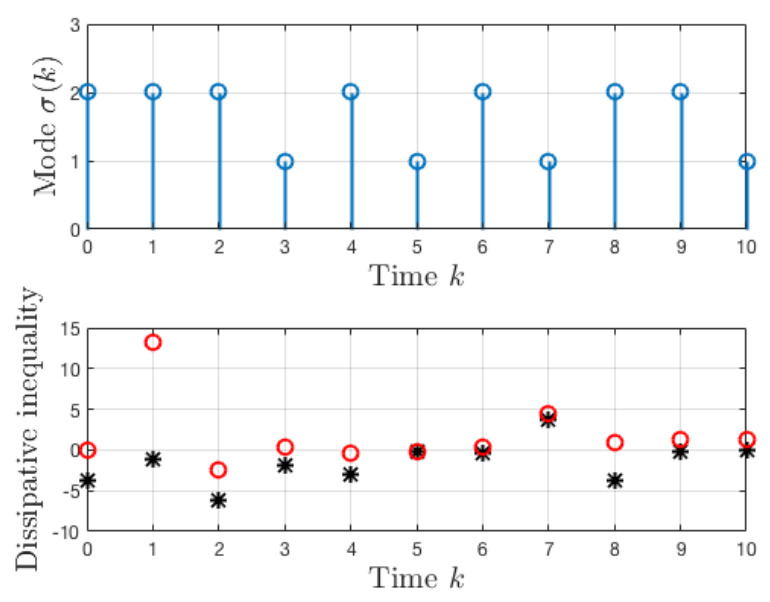

Fig. 2. Top: min-switching law $\sigma(k)=\mu\left(x_{k}\right)$ defined by (20); Bottom: current value of the supply rate (circle in red); $S_{\max }\left(x_{k+1}\right)-S_{\max }\left(x_{k}\right)$ (star in black).

\section{CONCLUSION}

The issue of dissipativeness for discrete-time linear switched systems has been investigated in this paper. Starting from the derivation of necessary and sufficient conditions in the time-dependent framework for dissipativeness, we obtain a concept of dissipativeness for a given switching law. Then, we assume that the supply rates are quadratic. That allows to establish our two main contributions. First, we provide sufficient conditions in terms of linear matrix inequalities ensuring that for any switching law, the discrete-time linear switched system is dissipative. The second contribution consists of providing parameterized linear matrix inequalities with parameters belonging to the entries of a Metzler matrix. That guarantees that there exists a min-switching law that renders the discrete-time linear switched system dissipative. Such a min-switching law is then build via multiple storage functions associated to multiple supply rates. These results have been discussed and illustrated in a numerical exemple.

\section{REFERENCES}

[1] J. C. Willems, "Dissipative dynamical systems part I: General theory," Archive for Rational Mechanics and Analysis, vol. 45, no. 5, pp. 321-351, 1972.
[2] — , "Dissipative dynamical systems," European Journal of Control, vol. 13, pp. 134-151, 2007.

[3] V. A. Yakubovich, "Solution of some matrix inequalities encountered in the automatic control theory," Doklady Akademii Nak, vol. 43, pp. 1304-1307, 1962.

[4] R. Kalman, "Lyapunov functions for the problem of Lur'e in automatic control," Proceedings of National Academy of Sciences, no. 49, pp. 201-205, 1963.

[5] A. I. Lur'e and V. N. Postnikov, "On the theory of stability of control systems," Applied Mathematics and Mechanics, vol. 8, no. 3, pp. 3-13, 1944.

[6] V. Popov, "Absolute stability of nonlinear systems of automatic control," Avtomatika i Telemekhanika, vol. 22(8), pp. 961-979, 1961.

[7] A. Rantzer, "On the Kalman-Yakubovich-Lemma," Systems \& Control Letters, vol. 28, no. 1, pp. 7-10, 1996.

[8] B. Brogliato, B. Maschke, R. Lozano, and O. Egeland, Dissipative Systems Analysis and Control. Springer London, 2007.

[9] L. Hitz and B. D. O. Anderson, "Discrete positive-real functions and their application to system stability," Proceedings of the Institution of Electrical Engineers, vol. 116, no. 1, pp. 153-155, 1969.

[10] A. Y. Pogromsky, M. Jirstrand, and P. Spångéus, "On stability and passivity of a class of hybrid systems," in Proceedings of the 37th IEEE Conference on Decision and Control, 1998, pp. 3705-3710.

[11] M. Žefran, F. Bullo, and M. Stein, "A notion of passivity for hybrid systems," in Proceedings of the 40th IEEE Conference on Decision and Control, vol. 1, 2001, pp. 768-773.

[12] J. Zhao and D. J. Hill, "A notion of passivity for switched systems with state-dependent switching," Journal of Control Theory and Applications, vol. 4, no. 1, pp. 70-75, 2006.

[13] - "Dissipativity theory for switched systems," IEEE Transactions on Automatic Control, vol. 53, no. 4, pp. 941-953, 2008.

[14] J. C. Geromel, P. Colaneri, and P. Bolzern, "Passivity of switched linear systems: Analysis and control design," Systems \& Control Letters, vol. 61, pp. 549-554, 2012.

[15] A. Bemporad, G. Bianchini, F. Brogi, and F. Barbagli, "Passivity analysis and passification of discrete-time hybrid systems," in Proceedings of the 16th IFAC World Congress, vol. 38, no. 1, 2005, pp. 391-396.

[16] Y. Wang, V. Gupta, and P. J. Antsaklis, "On passivity of a class of discrete-time switched nonlinear systems," IEEE Transactions on Automatic Control, vol. 59, no. 3, pp. 692-702, 2014.

[17] R. Naldi and R. G. Sanfelice, "Passivity-based control for hybrid systems with applications to mechanical systems exhibiting impacts," Automatica, vol. 49, no. 5, pp. 1104-1116, 2013.

[18] —, "Sufficient conditions for passivity and stability of interconnections of hybrid systems using sums of storage functions," in Proceedings of the 2014 American Control Conference, 2014, pp. 1432-1437.

[19] D. Hill and P. Moylan, "The stability of nonlinear dissipative systems," IEEE Transactions on Automatic Control, vol. 21, no. 5, pp. 708-711, 1976.

[20] S. S. Ge, Z. Sun, and T. H. Lee, "Reachability and controllability of switched linear discrete-time systems," IEEE Transactions on Automatic Control, vol. 46, no. 9, pp. 1437-1441, 2001.

[21] H. L. Trentelman and J. C. Willems, "Storage functions for dissipative linear systems are quadratic state functions," in Proceedings of the 36th IEEE Conference on Decision and Control, vol. 1, 1997, pp. 42-47.

[22] J. Daafouz, P. Riedinger, and C. Iung, "Stability analysis and control synthesis for switched systems : A switched Lyapunov function approach," IEEE Transactions on Automatic Control, vol. 47, pp. 1883-1887, 2002.

[23] J. C. Geromel and P. Colaneri, "Stability and stabilization of discrete-time switched systems," International Journal of Control, vol. 79, no. 7, pp. 719-728, July 2006.

[24] M. Fiacchini and M. Jungers, "Necessary and sufficient condition for stabilizability of discrete-time linear switched systems: a set-theory approach," Automatica, regular paper, vol. 50, no. 1, pp. 75-83, January 2014

[25] M. Fiacchini, A. Girard, and M. Jungers, "On the stabilizability of discrete-time switched linear systems: novel conditions and comparisons," IEEE Transactions on Automatic Control, Full paper, vol. 61, no. 5, pp. 1181-1193, 2016 